\title{
THE ONSET OF CONVECTION IN HORIZONTAL CYLINDERS
}

\author{
BY
}

JOHN P. MCHUGH

Department of Mechanical Engineering, University of New Hampshire, Durham, NH

\begin{abstract}
The convective instability of a fluid that fills a horizontal cylindrical cavity is considered. The boundaries of the cavity are conducting, and the driving force is a linear temperature gradient far from the cylinder. Critical Rayleigh numbers governing the onset of convection are determined for neutral stability. The results show that the critical Rayleigh number depends on the ratio of thermal conductivities of the solid to the fluid $(\lambda)$, and a wavenumber. Both two- and three-dimensional disturbances are included. The disturbances are separated into even modes and odd modes. The most unstable odd modes have been found to be two-dimensional, while the most unstable even modes are three-dimensional. The two-dimensional odd modes are most unstable in the vicinity of $\lambda=1$. The three-dimensional even modes are more unstable for other values of $\lambda$. The results are compared with the previous results of Gershuni and Zhukhovitskii.
\end{abstract}

1. Introduction. Consider a cavity in a solid which has the shape of a cylinder and is filled with a homogeneous fluid. The axis of the cylinder is oriented horizontally. The convective instability of the fluid is treated here. The driving force of the instability is a linear temperature gradient in the solid far from the cylinder.

The problem relates to many scientific and engineering problems. Of particular importance is the migration of fluid inclusions in a geological deposit where nuclear waste is stored. A fluid inclusion is a volume of fluid trapped in a solid. If the solid is soluble in the fluid, such as a water inclusion in a salt dome, the fluid inclusion will migrate through the solid when a temperature gradient is applied [10], [1], [8]. Nuclear waste is currently stored in salt domes at several locations around the world [9], [6]. Although sealed in glass to eliminate most of the harmful radiation, the nuclear waste still produces small amounts of heat. When stored in large quantities, there is sufficient energy to result in a temperature gradient in the surrounding geological material. If the fluid inclusions in the material become convectively unstable, the migration occurs much more rapidly. The inclusions can reach a fissure or other opening, and contribute to the transport of pollutants from the stored waste [5].

Received June 20, 1997.

2000 Mathematics Subject Classification. Primary 76E06, 76R10.

E-mail address: jpm@cisunix.unh.edu 
The fluid inclusions exist in many shapes. It is desirable to determine the region surrounding the waste chamber where an inclusion of any shape will become convectively unstable, thus providing an estimate of the quantity of fluid that could be affected by the waste. The cylindrical shape considered here represents an extreme version of an elongated inclusion, and may be the most unstable, thus giving the most conservative estimate.

The convective instability problem studied here was considered previously by Gershuni and Zhukhovitskii [2]. A review is provided in the book by the same authors [3]. The results of Gershuni and Zhukhovitskii predict unstable Rayleigh numbers, and incorrectly claim that two-dimensional odd disturbances are always more unstable than two- or threedimensional even disturbances. The method they used for the solution was an expansion using a low-order polynomial. Convergence of the expansion was not demonstrated.

The problem is now considered using the method of Yih [11]. Details are provided in McHugh [7]. The results here provide a more accurate value of the critical Rayleigh number, and show that three-dimensional even modes are sometimes more unstable than two-dimensional odd modes, disagreeing with Gershuni and Zhukhovitskii [2]. A comparison is made between the present results and similar results in a rectangular geometry by Hurle [4] and a spherical geometry by Yih [11].

2. Primary quantities. Consider a horizontal circular cylinder of fluid embedded in a solid, and let the cylinder have radius, $a$, and an infinite length. The axis of the cylinder is coincident with the $z$-axis, oriented horizontally. Figure 1 shows the coordinate system.

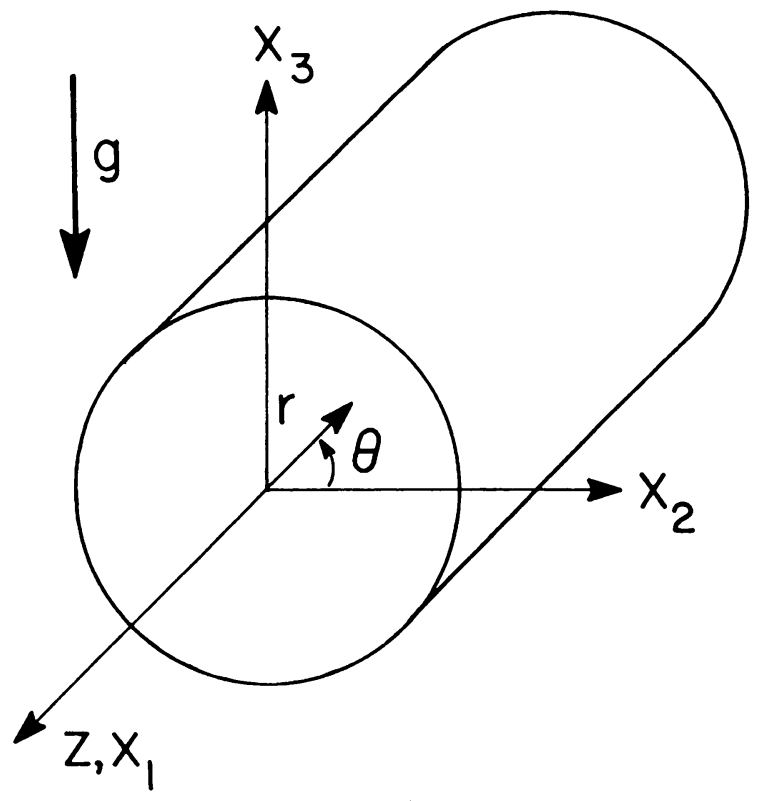

FIG. 1. Geometry and coordinate system 
The driving force leading to instability is a temperature gradient which is assumed to be linear far from the cylinder. The primary state assuming no motion was given by Yih $[10]:$

$$
\begin{gathered}
\bar{T}_{s}=\beta_{s} x_{3}+\beta_{1} a^{2} \frac{x_{3}}{r^{2}}+T_{0}, \\
\bar{T}=\beta x_{3}+T_{0},
\end{gathered}
$$

where

$$
\begin{aligned}
\beta_{s} & =\frac{\beta}{2}\left(\frac{\lambda+1}{\lambda}\right), \\
\beta_{1} & =\frac{\beta}{2}\left(\frac{\lambda-1}{\lambda}\right), \\
\lambda & =\frac{k_{s}}{k}
\end{aligned}
$$

$T_{0}$ is a reference temperature, $k$ is the conductivity, $r$ is the radial coordinate, and $x_{3}$ is the distance parallel to gravity. Note that quantities in the solid are denoted with the subscript, $s$, and the overbar indicates a primary variable.

3. Solution for two-dimensional disturbances. The governing equations are the Navier-Stokes equations, continuity, and the energy equation, which are made dimensionless using $a$ as the length scale, $\kappa / a^{2}$ as the time scale, and $\beta a$ as the temperature scale, where $\kappa$ is the diffusivity of the fluid. All variables are separated into a primary and a disturbance part, and products of the disturbance quantities are neglected. The resulting linear disturbance equations are

$$
\begin{gathered}
\frac{\partial \widehat{T}}{\partial t}+u_{3}=\nabla^{2} \widehat{T}, \\
\frac{\partial \widehat{T}_{s}}{\partial t}=\lambda \nabla^{2} \widehat{T}_{s}, \\
\frac{1}{P_{r}} \frac{\partial u_{i}}{\partial t}=-\frac{\partial \hat{p}}{\partial x_{i}}-R \widehat{T} \delta_{i 3}+\nabla^{2} u_{i}, \\
\frac{\partial u_{i}}{\partial x_{i}}=0,
\end{gathered}
$$

where the circumflex indicates a disturbance quantity, and

$$
P_{r}=\frac{\nu}{\kappa}, \quad R=-\frac{g \alpha \beta a^{4}}{\kappa \nu}
$$

are the Prandtl and Rayleigh numbers, respectively.

The boundary conditions at the cylindrical surface are zero velocity, continuity of temperature, and continuity of heat flux, which demand that

$$
u_{i}=0, \quad \widehat{T}=\widehat{T}_{s}, \quad \frac{\partial \widehat{T}}{\partial r}=\lambda \frac{\partial \widehat{T}_{s}}{\partial r},
$$


on $r=1$, respectively. Furthermore, $\widehat{T}_{s} \rightarrow 0$ as $r \rightarrow \infty$, and $\widehat{T}, u_{i}$, and $\hat{p}$ must be regular at $r=0$.

Neutral stability is assumed and the derivatives with respect to time are deleted (see Yih's [11] proof of the exchange of stabilities). The solution is found by expanding $h$ using

$$
h=\sum_{n=0}^{\infty} \sum_{j=1}^{\infty} A_{n j} J_{n}\left(\alpha_{n j} r\right) \cos n \theta
$$

where $h=R \widehat{T}, J_{n}$ are Bessel functions of the first kind of integer order, $A_{n j}$ are constants chosen by the initial conditions, and $\alpha_{n j}$ are constants that are determined by the boundary conditions on temperature. The linear equations are then solved for $u_{3}$. The details are tedious and are provided in McHugh [7]. The final solution is

$$
\begin{aligned}
u_{3}=\sum_{n=0}^{\infty} \sum_{j=1}^{\infty} A_{n j}\left(\frac { 1 } { 4 \alpha _ { n j } ^ { 2 } } \left[-\left[4-\left(a_{n}+b_{n}\right)\right] J_{n} \cos n \theta+a_{n} J_{n+2} \cos (n+2) \theta\right.\right. & \left.+b_{n} J_{n-2} \cos (n-2) \theta\right] \\
+ & \frac{1}{4} B_{n j} r^{n+2} \cos n \theta+\frac{1}{4} C_{n j} r^{n} \cos (n-2) \theta \\
+ & \left.F_{n j} r^{n} \cos n \theta+G_{n j} r^{n+2} \cos (n+2) \theta+H_{n j} r^{n-2} \cos (n-2) \theta\right)
\end{aligned}
$$

where

$$
\begin{gathered}
a_{n}= \begin{cases}2 & n=0, \\
1 & n>0,\end{cases} \\
b_{n}= \begin{cases}0 & n=0,1, \\
1 & n>1 .\end{cases}
\end{gathered}
$$

Note that the argument of the Bessel functions in (13) is $\alpha_{n j} r$. The five constants are evaluated with the boundary conditions.

4. Three-dimensional disturbances. Consider the same primary state, but now let the disturbances be three-dimensional. The procedure for finding the eigenvalues is essentially the same as the two-dimensional case. The expansion for $h$ is

$$
h=\sum_{n=0}^{\infty} \sum_{j=1}^{\infty} A_{n j} J_{n}\left(\alpha_{n j} r\right) \cos n \theta \cos k z .
$$


The resulting solution is

$$
\begin{aligned}
u_{3}=\sum_{n=0}^{\infty} \sum_{j=1}^{\infty} A_{n j}(- & \frac{4 k^{2}+\left[4-\left(a_{n}+b_{n}\right)\right] \alpha_{n j}^{2}}{4\left(\alpha_{n j}^{2}+k^{2}\right)^{2}} J_{n} \cos n \theta \\
& +\frac{\alpha_{n j}^{2}}{4\left(\alpha_{n j}^{2}+k^{2}\right)^{2}}\left[a_{n} J_{n+2} \cos (n+2) \theta+b_{n} J_{n-2} \cos (n-2) \theta\right] \\
& +B_{n j} \frac{1}{4 k}\left[r I_{n}^{\prime} \cos n \theta-r I_{n+2}^{\prime} \cos (n+2) \theta\right] \\
& -C_{n j} \frac{1}{4 k}\left[r I_{n}^{\prime} \cos n \theta-r I_{n-2}^{\prime} \cos (n-2) \theta\right] \\
& +F_{n j} I_{n} \cos n \theta+G_{n j} I_{n+2} \cos (n+2) \theta \\
& \left.+H_{n j} I_{n-2} \cos (n-2) \theta\right) \cos k z
\end{aligned}
$$

where $I_{n}$ is the hyperbolic Bessel function. Note that the argument of the hyperbolic Bessel functions is $k r$.

5. Evaluation of the critical Rayleigh number. The value of the Rayleigh number for marginal stability is calculated with either the two-dimensional or three-dimensional theory using the reduced form of the heat equation:

$$
\nabla^{2} h=R u_{3} .
$$

The two-dimensional results are obtained by inserting (12) and (13) into (18). The coefficient of $\cos n \theta$ is identified for $n=0,1,2, \ldots$, multiplied by $r J_{n}\left(\alpha_{n l} r\right)$, and integrated from zero to unity for $l=1,2,3, \ldots$. The result is an infinite set of algebraic equations in the coefficients, $A_{n j}$. Taking a finite number of coefficients, the determinant of the coefficient matrix determines the value of $R$. The procedure for the three-dimensional case is to insert (16) and (17) into (18), then proceed as above.

The algebra involved in taking the determinant of the coefficient matrix for more than two coefficients is unmanageable. Furthermore, it has been found that the results are not converged with only two coefficients. Therefore, the value of the critical $R$ is evaluated numerically for an arbitrary number of coefficients. There are two steps to the numerical part of the work. The first step is to determine $\alpha_{n j}$ for a chosen value of $\lambda$ (and $k$ for the three-dimensional theory). The values of $\alpha_{n j}$ are found numerically such that the expansion for $h$ satisfies the temperature boundary condition. Note that this is the only place in the numerical work where $\lambda$ is involved.

It is interesting to compare the two-dimensional results to the three-dimensional results. As $k \rightarrow 0$, the three-dimensional case is equivalent to the two-dimensional case. However, the values of $\alpha_{n j}$ for the two-dimensional case do not match the values for three dimensions as $k \rightarrow 0$. They are different sets of numbers. Yet, the values of the critical Rayleigh number for the three-dimensional case with $k \rightarrow 0$ do match the twodimensional results to an arbitrary number of digits. Furthermore, the analytic solutions 
determined previously for three dimensions do not reduce to those for two dimensions as $k \rightarrow 0$.

The second step in the calculation is the determination of the critical value of $R$. This is accomplished by forming a matrix equation out of (18) in the following form:

$$
[A+R B]\left(A_{n j}\right)=0,
$$

where $A$ and $B$ are matrices. The matrix $A$ is inverted and the equation is left-multiplied by $A^{-1}$ to get

$$
\left[A^{-1} B+\frac{1}{R} I\right]\left(A_{n j}\right)=0 \text {. }
$$

The values of $\frac{1}{R}$ are determined using the $Q R$ method, and then inverted to obtain the critical Rayleigh numbers.

The solution can be split into even and odd modes, with regard to a vertical plane of symmetry along the center of the cylinder. Thus an even mode has $u\left(x_{2}\right)=u\left(-x_{2}\right)$, and an odd mode has $u\left(x_{2}\right)=-u\left(-x_{2}\right)$. The calculation for $R$ is performed for the even and odd modes separately, for an arbitrary set of $n$ 's and $j$ 's. The maximum value of each index is chosen independently. Convergence is realized when the value of $R$ ceases to change with an increase in either index. Table 1 shows that convergence is obtained quite rapidly with this method. The values in Table 1 are for three-dimensional odd modes with $\lambda=1$ and $k=1$. Convergence to six decimal places is obtained for these modes with only two values of $n(n=1$ and 3$)$ and ten values of $j$. Note that the convergence with increasing $n$ is substantially faster than with increasing $j$. Convergence with other modes was similar.

TABLE 1. Convergence of the critical Rayleigh number for $k=1$ and $\lambda=1$

\begin{tabular}{cc|ccccccc} 
& \multicolumn{8}{|c}{$j_{\max }$} \\
$r_{\max }$ & 1 & 2 & 3 & 4 & 5 & 10 & 20 \\
\hline & 1 & 289.454 & 276.797 & 276.253 & 276.175 & 276.156 & 276.147 & 276.147 \\
& 3 & 289.345 & 276.612 & 276.071 & 275.993 & 275.975 & 275.965 & 275.965 \\
& 5 & 289.345 & 276.612 & 276.071 & 275.993 & 275.975 & 275.965 & 275.965 \\
& 7 & 289.345 & 276.612 & 276.071 & 275.993 & 275.975 & 275.965 & 275.965
\end{tabular}

6. Results. Figure 2 shows the critical Rayleigh number versus $\lambda$ for the two-dimensional theory. Only the most unstable even and odd modes are shown. There is actually an infinite set of modes, all with greater values of $R$ than those shown in Fig. 2. The higher-order modes that have been studied have the same trend as the two modes shown. Note in Fig. 2 that the odd modes are always more unstable than the even modes. Also, note the effect of $\lambda$, which is that $R$ increases monotonically with $\lambda$, finally reaching an asymptotic value. These trends (with two-dimensional disturbances) match the previous results of Yih [11] for the spherical geometry and Hurle, et. al. [4] for the rectangular geometry. 


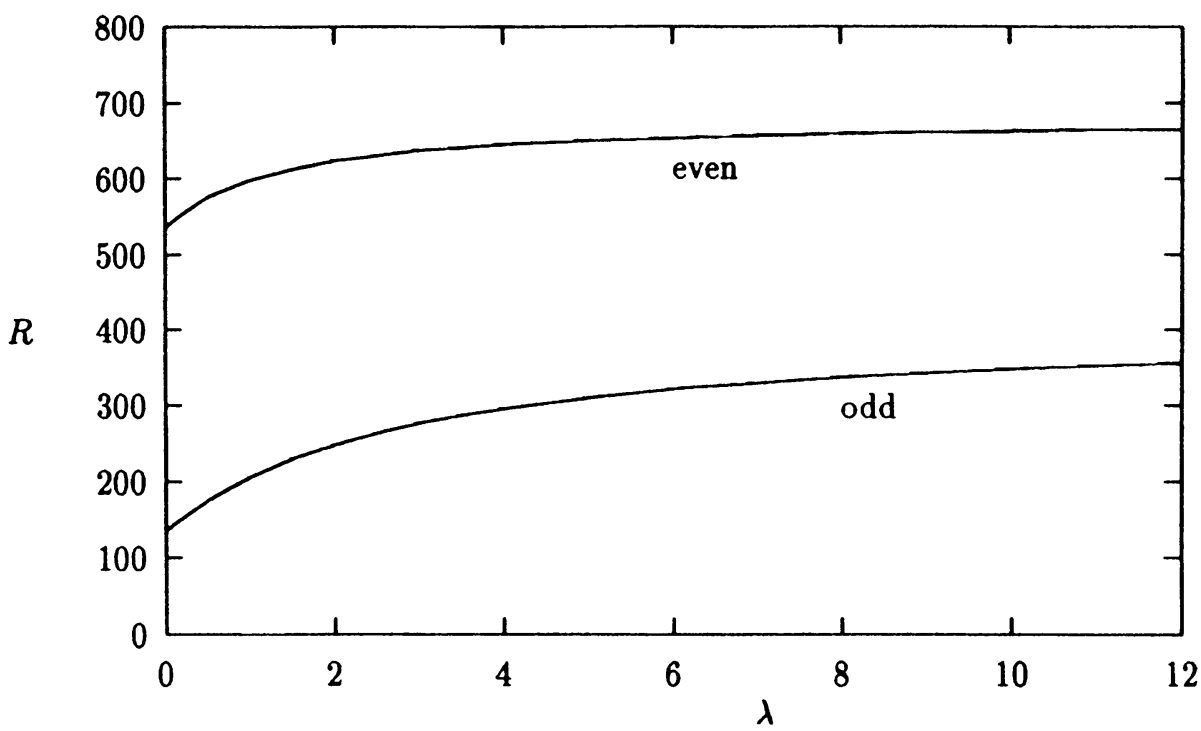

FIG. 2. Critical Rayleigh number $\left(R=-\frac{g \alpha \beta a^{4}}{\kappa \gamma}\right)$ versus $\lambda\left(=\kappa_{s} / \kappa\right)$ for two-dimensional disturbances

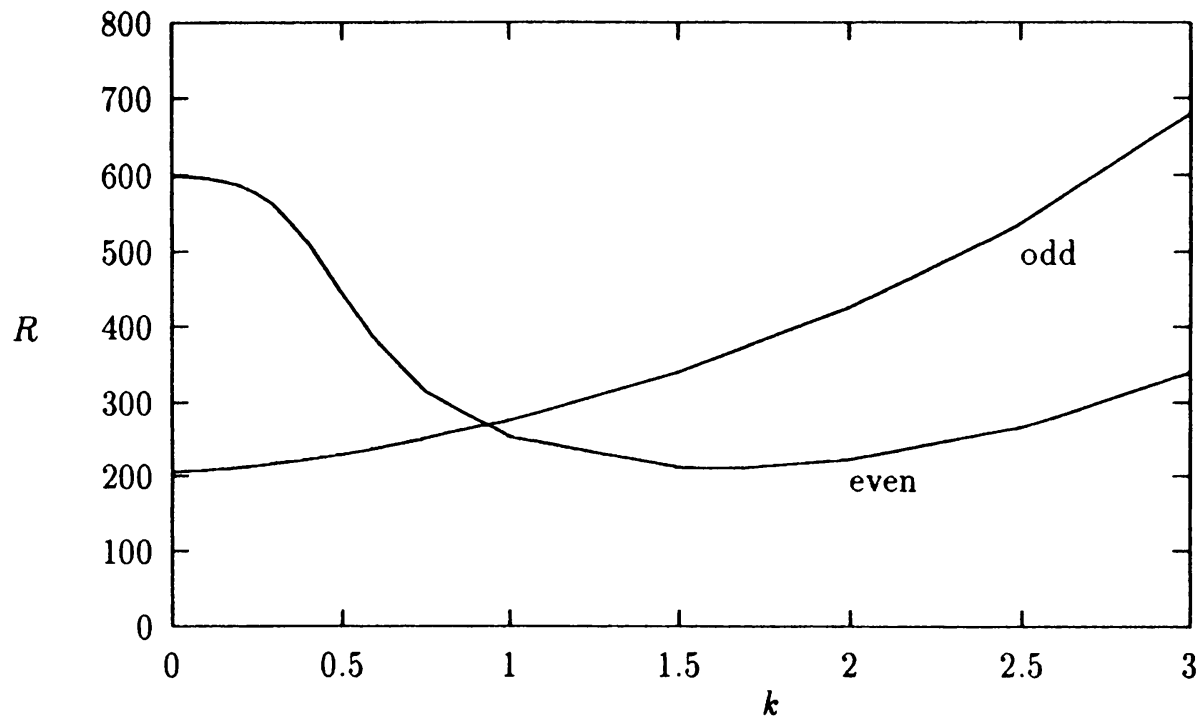

FIG. 3. Critical Rayleigh number $\left(R=-\frac{g \alpha \beta a^{4}}{\kappa \gamma}\right)$ versus $k$ for threedimensional disturbances with $\lambda=\kappa_{s} / \kappa=1$

The results for the three-dimensional theory depend on the axial wavenumber, $k$, as well as $\lambda$. Figure 3 shows critical Rayleigh numbers for the three-dimensional theory versus $k$. Again, only the most unstable odd mode and the most unstable even mode are shown. 
The odd modes are seen to have a minimum value at zero $k$, which corresponds to a two-dimensional disturbance. The minimum value for $\lambda=1$ is 206.18. The even modes in Fig. 3 attain a minimum value of the critical Rayleigh number for a nonzero value of $k$. For the case of $\lambda=1$, the minimum is attained at $k=1.605$, and the corresponding minimum Rayleigh number is 210.63 . This minimum value does depend on $\lambda$, as will be seen.

The odd mode has the lowest value of $R$ for $\lambda=1$ (Fig. 3); therefore, the odd mode is more unstable. However, for other values of $\lambda$, the even mode may be more unstable. The most unstable mode for the odd case has been found to always be the two-dimensional case. The most unstable mode for the even case must be determined by finding the minimum in the relation between $R$ and $k$. The most unstable odd and even modes versus $\lambda$ are shown in Fig. 4 . The values of $k$ that correspond to the most unstable even modes are shown in Fig. 5.

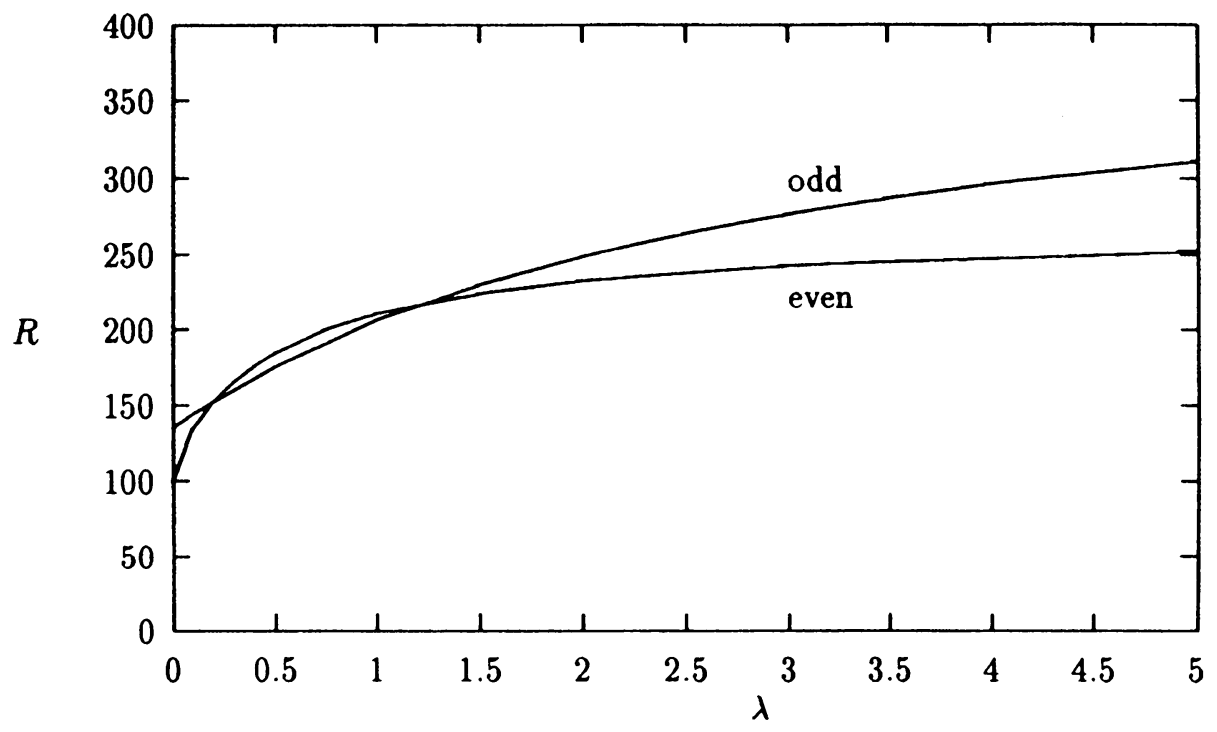

FIG. 4. Critical Rayleigh number $\left(R=-\frac{g \alpha \beta a^{4}}{\kappa \gamma}\right)$ versus $\lambda\left(=\kappa_{s} / \kappa\right)$ for the most unstable even and odd modes

Figure 6 shows isotherms from the eigenvectors of the disturbance solution for the most unstable even (Fig. 6a) and odd (Fig. 6b) modes. These temperature patterns are different than the streamline patterns. The odd modes show motion in a single cell with streamlines that are closed loops surrounding the origin. The even modes have a vertical streamline through the center of the cylinder, and two mirror image cells on either side. The difference between the temperature patterns and velocity patterns indicates that conduction and convection are comparable in magnitude in the disturbance solution.

Note in Fig. 4 that sometimes the even modes are more unstable, and sometimes the odd modes are more unstable, depending on the ratio of conductivities, $\lambda$. Odd modes are most unstable in the vicinity of $\lambda=1$, and even modes are most unstable for other values of $\lambda$. This result disagrees with Hurle, et. al., [4] and Yih [11]. Hurle, et. al., [4] 


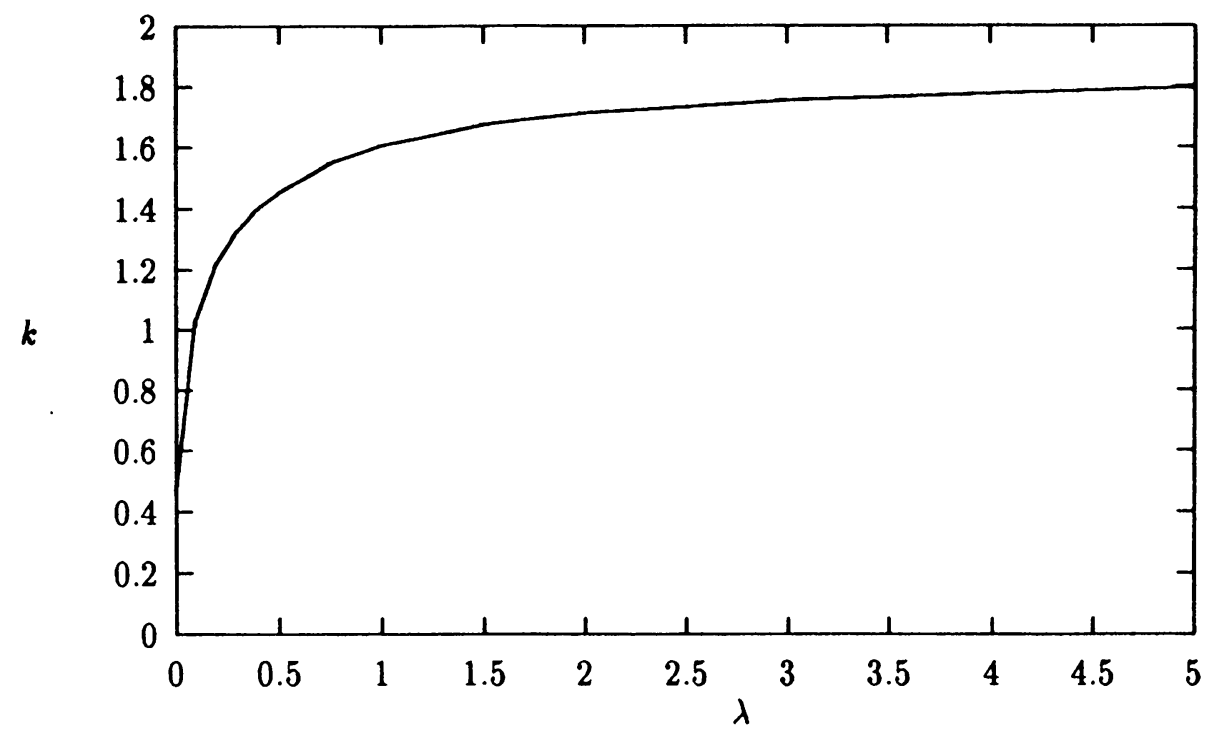

FIG. 5. Wavenumber for the most unstable even mode

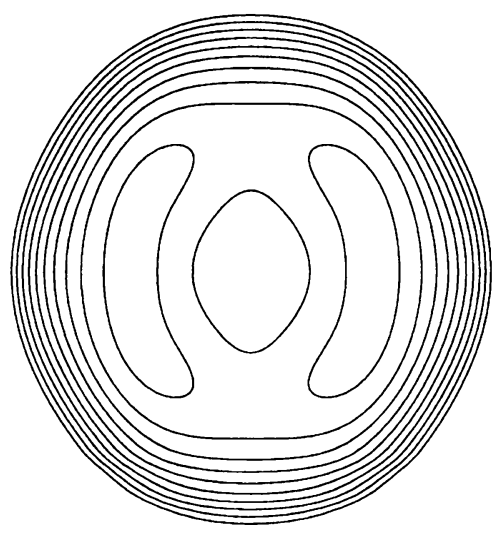

FIG. 6a. Isotherms for the even disturbances with $\lambda=\kappa_{s} / \kappa=1$

showed that even modes are always more unstable than odd modes for the rectangular geometry. Yih [11] showed the opposite trend for the spherical geometry; odd modes are always more unstable than even modes.

One can speculate about the competition between even and odd modes in confined geometries with conducting boundaries. The sphere of Yih [11] had confined boundaries in all horizontal directions, and odd modes were most unstable. The rectangular shape of Hurle, et. al. [4] was unbounded in the horizontal direction, and even modes are most unstable. The cylinder considered here has one bounded direction and one unbounded direction, and the most unstable mode may be even or odd. The speculation is that geometries with unbounded horizontal directions favor even modes, while bounded 


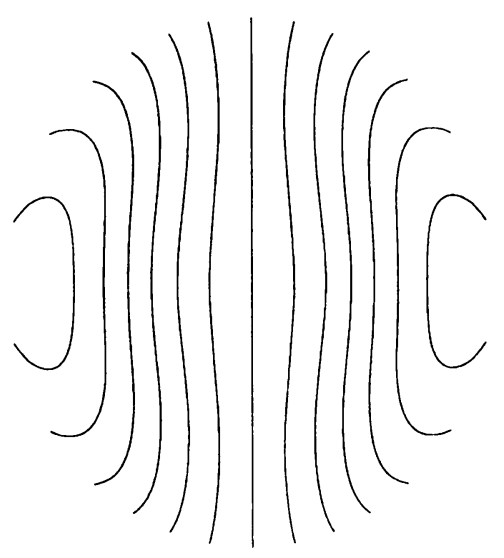

FIG. 6b. Isotherms for the odd disturbances with $\lambda=\kappa_{s} / \kappa=1$

geometries favor odd modes. For example, one might hypothesize that a geometry consisting of an ellipse of revolution will be more unstable to odd modes rather than even modes.

As discussed previously, Gershuni and Zhukhovitskii [2] considered the same problem. They expanded all variables with

$$
f=\left(1-r^{2}\right) \sum_{m=0}^{M} \sum_{n=0}^{N} A_{n m} x_{2}^{m} x_{3}^{n},
$$

where $f$ represents any of the dependent variables. The order of the polynomials was restricted according to

$$
M+N \leq 2
$$

so that analytic results could be obtained.

A direct comparison between the present results and those of Gershuni and Zhukhovitskii [2] is given in Table 2. Gershuni and Zhukhovitskii use the parameter, $\alpha$, which is merely the inverse of $\lambda$. Note that $\frac{1}{\lambda}$ is listed along with $\lambda$ in Table 2, for convenience. The present results differ substantially from the older results. For large $\lambda$ the difference is nearly a factor of two. The two sets of results match best near $\lambda$ of unity.

TABLE 2. Comparison with Gershuni and Zhukhovitskii of critical Rayleigh numbers

\begin{tabular}{cc|cc|cc} 
& & \multicolumn{2}{|c|}{ Odd modes } & \multicolumn{2}{c}{ Even modes } \\
$\lambda$ & $\frac{1}{\lambda}$ & Gershuni & Present & Gershuni & Present \\
\hline 0.01 & 100 & 102 & 136.19 & 138 & 105.77 \\
0.1 & 10 & 134 & 144.37 & 147 & 134.49 \\
1 & 1 & 210 & 206.18 & 213 & 210.63 \\
$\infty$ & 0 & 260 & 406.76 & 480 & 268.62
\end{tabular}

The present results have been shown to be converged as terms are added to the expansions. Gershuni and Zhukhovitskii apparently could not perform such a test, due 
to the reliance on a purely analytic result. Furthermore, the choice of simple polynomials by Gershuni and Zhukhovitskii is not expected to be as accurate as the Bessel function expansions used here, given the Bessel operators which appear in the governing equations. Therefore the present results are believed to be correct.

The even modes as $k$ approaches zero show a different trend than that presented by Gershuni and Zhukovitskii [2]. This is evident in Fig. 7, which shows the same two modes as Fig. 3, plus two more modes, one even and one odd. Thus the modes shown in

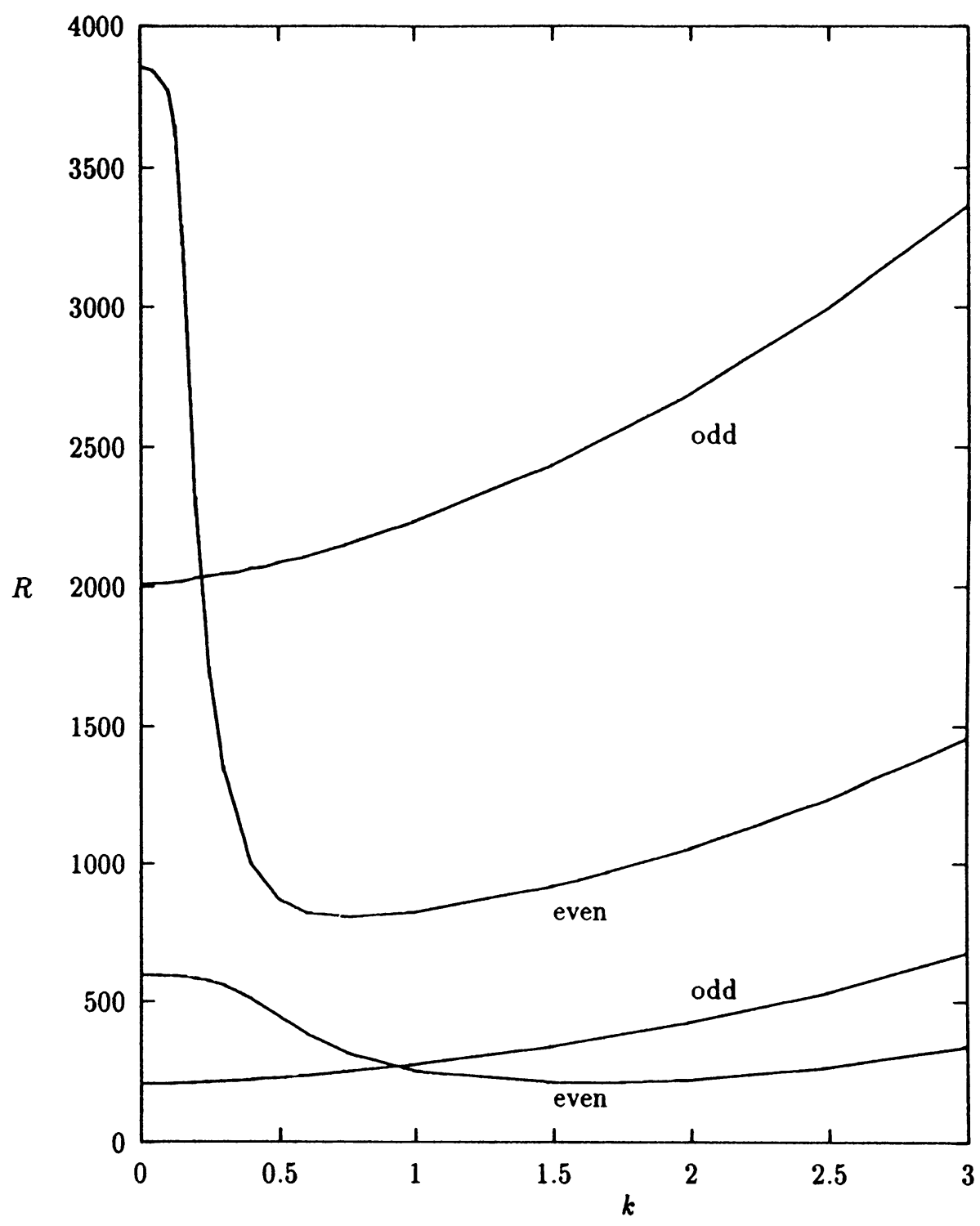

FIG. 7. Critical Rayleigh number $\left(R=-\frac{g \alpha \beta a^{4}}{\kappa \gamma}\right)$ for threedimensional disturbances for several higher modes, with $\lambda=\kappa_{s} / \kappa=1$ 
Fig. 7 are the four most unstable modes for $\lambda=1$. The present results show that as $k$ approaches zero, the slope of the curve also approaches zero. Gurshuni and Zhukhovitskii show a nonzero slope for the first even mode, and then claim that the second even mode is singular at $k=0$. The results of Gershuni and Zhukhovitskii are believed to suffer excessive truncation error in a region of small $k$, and the present results are again correct.

Figure 7 also shows that the second odd mode has a minimum at $k=0$, and the second even mode reaches a minimum at a nonzero value of $k$, as do the first two modes. Note that the second even mode is much more unstable than the second odd mode, unlike the first modes at $\lambda=1$.

Acknowledgment. The author would like to acknowledge the late Professor ChiaShun Yih for suggesting this problem and for many helpful discussions.

\section{REFERENCES}

[1] T. R. Anthony and H. E. Cline, Thermal migration of liquid droplets through solids, Journal of Applied Physics 42, 3380-3387 (1971)

[2] G. Z. Gershuni and E. M. Zhukhovitskii, The stability of equilibrium of fluid within a horizontal cylinder heated from below, J. Appl. Math. Mech. 25, 1551-1558 (1961)

[3] G. Z. Gershuni and E. M. Zhukhovitskii, Convective instability of incompressible fluids, Jeter Press, 1976

[4] D. T. J. Hurle, E. Jakeman, and E. R. Pike, On the solution of the Bénard problem with boundaries of finite conductivity, Proc. Royal Soc. London Ser. A 296, 469-475 (1967)

[5] Y. Hwang, T. H. Pigford, P. L. Chambre, and W. Lee, Analysis of mass transport in a nuclear waste repository in salt, Water Resources Research 28, 1857-1868 (1992)

[6] J. I. Kim, K. Gompper, K. D. Closs, G. Kessler, and D. Faude, German approaches to closing the nuclear fuel cycle and final disposal of HLW, Journal of Nuclear Materials 238, 1-10 (1996)

[7] J. P. McHugh, Notes on the onset of convection in horizontal cylinders, FPR report 101, 1998

[8] D. R. Olander, A. J. Machiels, M. Balooch, and S. K. Yagnik, Thermal gradient migration of brine inclusions in synthetic alkali halide single crystals, Journal of Applied Physics 53, 669-681 (1982)

[9] W. Xu and J. Genin, Closure of a nuclear waste repository deeply imbedded in a stratified salt bed, Journal of Engineering Materials and Technology 116, 567-573 (1994)

[10] C. S. Yih, Movement of liquid inclusions in soluble solids: An inverse Stokes' law, Physics of Fluids 29, 2785-2787 (1986)

[11] C. S. Yih, Convective instability of a spherical liquid inclusion, Physics of Fluids 30, 36-44 (1987) 